\title{
Semi-Invariant Form of Equilibrium Stability Criteria for Systems with One Cosymmetry
}

\author{
L. G. Kurakin, A. V. Kurdoglyan
}

The systems of differential equations with one cosymmetry are considered [1]. The ordinary object for such systems is a one-dimensional continuous family of equilibria. The stability spectrum changes along this family, but it necessarily contains zero. We consider the nondegeneracy condition, thus the boundary equilibria separate the family on linearly stable and instable areas. The stability of the boundary equilibria depends on nonlinear terms of the system.

The stability problem for the systems with one cosymmetry is studied in [2]. The general problem is to apply the stability criteria one needs to compute coefficients of the model system. It is especially difficult if the system has a large dimension, while a number of critical variables may be small. A method for calculating coefficients is proposed in [3].

In this work the expressions for the known stability criteria are proposed in a form convenient for calculation. The explicit formulas of the coefficients of the model system are given in semi-invariant form. They are expressed using the generalized eigenvectors of the linear matrix and its conjugate matrix.

Keywords: stability, critical case, neutral manifold, cosymmetry, semi-invariant form

Received July 15, 2019

Accepted November 05, 2019

This work was supported by the Ministry of Education and Science of the Russian Federation, Southern Federal University (Projects No. 1.5169.2017/8.9).

Leonid G. Kurakin

kurakin@math.rsu.ru

Southern Mathematical Institute, Vladikavkaz Scienific Center of RAS

ul. Markusa 22, Vladikavkaz, 362027 Russia

Water Problems Institute of RAS

ul. Gubkina 3, Moscow, 119333 Russia

Aik V. Kurdoglyan

aik_kurdoglyan@mail.ru

Southern Federal University

ul. Milchakova 8a, Rostov-on-Don, 344090 Russia

RUSSIAN JOURNAL OF NONLINEAR DYNAMICS, 2019, 15(4), 525-531 


\section{Formulation of the problem and description of the method}

An autonomous real differential equation in $\mathbb{R}^{n}$ with zero equilibrium is considered

$$
\dot{u}=f(u)=A u+g(u), \quad g(u)=\sum_{j=2}^{\infty} K_{j} u^{j}, \quad f(0)=0 .
$$

Here, $u \rightarrow f(u)$ is an analytical vector field defined in a neighborhood $\Omega$ of the point $0 \in \mathbb{R}^{n}$, and $A: \mathbb{R}^{n} \rightarrow \mathbb{R}^{n}$ is a linear operator. The operator $K_{j} u^{j}$ is symmetric and homogeneous of degree $j$. The operator $K_{j} u^{j}$ acting in $\mathbb{R}^{n}$ is defined by a $j$-linear mapping $\left(u_{1}, \ldots, u_{j}\right) \rightarrow$ $K_{j}\left(u_{1}, \ldots, u_{j}\right)$, and $K_{j} u^{j}=K_{j}(u, \ldots, u) ; u_{1}, \ldots, u_{j}, u \in \mathbb{R}^{n}$.

The map $f$ has one continuous cosymmetry $L: \Omega \rightarrow \mathbb{R}^{n}$. According to the definition introduced by V. I. Yudovich [1], this means that vectors $f(u)$ and $L(u)$ are orthogonal for each point $u \in \Omega$

$$
(f(u), L(u))=0 .
$$

We assume that the following assumptions are fulfilled.

Assumption 1. Zero equlibrium is not cosymmetric:

$$
L(0) \neq 0 .
$$

In [1] it is shown that vector $L(0)$ belongs to the kernel of the operator $A^{*}$ conjugated to the derivative $A=f^{\prime}(0)$. Thus, the zero belongs to the spectrum $\sigma(A)$ and $\sigma\left(A^{*}\right)$ of operators $A$ and $A^{*}$.

Assumption 2. The condition of degeneration minimality is fulfilled: the kernel of operator $A$ is one-dimensional: $\operatorname{dim} \operatorname{ker} A=1$.

Assumption 3. The spectrum $\sigma(A)$ of operator $A$ consists of a neutral set $\sigma_{0}(A)$ and a stable set $\sigma_{1}(A)$ :

$$
\sigma_{0}(A)=\{\lambda: \lambda \in \sigma(A), \operatorname{Re} \lambda=0\}, \quad \sigma_{1}(A)=\{\lambda: \lambda \in \sigma(A), \operatorname{Re} \lambda<0\} .
$$

The spectral subspaces $U_{0}, U_{1}$ and the spectral projectors $P_{0}, P_{1}$ correspond to the spectral sets $\sigma_{0}$ and $\sigma_{1}$.

According to [4], the invariant submanifold $M_{0} \subset V$ of the system (1.1) that touches the neutral subspace $U_{0}$ at the point $u=0$ exists in some neighborhood $V \subset \Omega \subset \mathbb{R}^{n}$ of the point $u=0$. The submanifold $M_{0}$ is a graph of some map $F: V_{0} \rightarrow U_{1}$, where $V_{0}=V \cap U_{0}$.

The set $M_{0}$ is sometimes called a neutral (or central) manifold.

It follows from the reduction principle [4] that the stability problems of zero equilibria of the original equation (1.1) and its reduction to $M_{0}$ are equivalent.

The system (1.1) is rewritten as

$$
\begin{aligned}
& \dot{u}_{0}=P_{0} f\left(u_{0}+u_{1}\right)=A_{0} u_{0}+P_{0} g\left(u_{0}+u_{1}\right), \\
& \dot{u}_{1}=P_{1} f\left(u_{0}+u_{1}\right)=A_{1} u_{1}+P_{1} g\left(u_{0}+u_{1}\right),
\end{aligned}
$$

where

$$
u_{k}=P_{k} u, \quad A_{k}=P_{k} A, \quad k=0,1 .
$$

Let us change the variables

$$
\xi_{0}=u_{0}, \quad \xi_{1}=u_{1}-F\left(u_{0}\right) .
$$


The neutral manifold is defined by the condition $\xi_{1}=0$. Equation (1.2) is rewritten in the form

$$
\begin{aligned}
& \dot{\xi}_{0}=P_{0} f\left(\xi_{0}+\xi_{1}+F\left(\xi_{0}\right)\right), \\
& \dot{\xi}_{1}=\left(P_{1}-F^{\prime}\left(\xi_{0}\right) P_{0}\right) f\left(\xi_{0}+\xi_{1}+F\left(\xi_{0}\right)\right) .
\end{aligned}
$$

According to the reduction principle, it suffices to investigate the stability of zero equilibrium on a neutral manifold $\left(\xi_{1}=0\right)$ :

$$
\dot{\xi}_{0}=P_{0} f\left(\xi_{0}+F\left(\xi_{0}\right)\right) .
$$

The map $F$ can be searched for as a Taylor series:

$$
F\left(\xi_{0}\right)=\sum_{j=2}^{\infty} F_{j} \xi_{0}^{j},
$$

where $F_{j}: \xi_{0} \rightarrow F_{j} \xi_{0}^{j} \quad(j=2,3, \ldots)$ is a symmetric vector-valued form of $j$ th degree on $U_{0}$ with values in $U_{1}$.

It is known that the series (1.5) may be divergent, but in each of the cases under consideration it is sufficient to know only a few of its first terms.

To determine $F$, we obtain from the invariance condition

$$
P_{1} f\left(\xi_{0}+F\left(\xi_{0}\right)\right)=F^{\prime}\left(\xi_{0}\right) P_{0} f\left(\xi_{0}+F\left(\xi_{0}\right)\right) .
$$

Substituting a series (1.5) into the last equation and equating in both parts terms of equal powers, we deduce the system of equations to determine forms $F_{j}(j=2,3, \ldots)$.

The change $v_{0}=\xi_{0}-G\left(\xi_{0}\right)\left(G: V_{0} \rightarrow U_{0}\right)$ leads Eq. (1.4) to normal form to some order [5].

The reduction of Eqs. (1.4) to normal form is simplified in the number of cases if $F$ and $G$ are searched for in the form $F=P_{1} T, G=P_{0} T$, and map $T: V_{0} \rightarrow V$ is defined so that the change of variables $v=u-T\left(u_{0}\right)$ in Eq. (1.3) leads to the fact that nonresonant terms containing $u_{0}$ vanish.

Thus, the stability problem of the zero equilibrium of Eq. (1.1) reduces to studying the equation on a neutral manifold in the normal form, which was studied in [2]. In this paper, the problem is classified according to the codimension of the degeneracy in the space of all possible cosymmetric systems.

Further, the results of [2] are presented in a semi-invariant form. The Jordan basis is used not in the whole space, but only on the neutral subspace. In these formulas, the dimension of the system does not appear anywhere, so they are also applicable for infinite-dimensional problems, for example, for systems of partial differential equations.

In each of the critical cases discussed below, the following scheme of presenting results is used:

1. Conditions of degeneration of the linear part are given. The neutral spectrum $\sigma_{0}$ is determined and the projector $P_{0}$ is introduced.

2. An equation on the neutral manifold $M_{0}$ in the normal form is given. An asymptotic representation for $\left|v_{0}\right| \rightarrow 0$ is used.

3. A model system is provided. Only those of the terms of the expansion of equations for the neutral manifold that participate in the stability criteria are written. A semi-invariant form of the stability criteria obtained in [2] is given. 


\section{The critical case of the triple zero eigenvalue}

1. The neutral spectrum is $\sigma_{0}(A)=\left\{0^{3}\right\}$. The eigenvalue is 0 of multiplicity 3 , which corresponds to the three-dimensional Jordan block. The corresponding generalized eigenvectors of operators $A$ and $A^{*}$ are $\varphi_{1}, \varphi_{2}, \varphi_{3}$ and $\Phi_{1}=L(0), \Phi_{2}, \Phi_{3}$ :

$$
\begin{gathered}
A \varphi_{1}=0, \quad A \varphi_{2}=\varphi_{1}, \quad A \varphi_{3}=\varphi_{2}, \quad A^{*} \Phi_{1}=0, \quad A^{*} \Phi_{2}=\Phi_{1}, \quad A^{*} \Phi_{3}=\Phi_{2} ; \\
\left(\varphi_{j}, \Phi_{4-j}\right)=1, \quad\left(\varphi_{k}, \Phi_{l}\right)=0, \quad j, k, l=1,2,3 ; \quad k+l \neq 4 .
\end{gathered}
$$

The projector on the neutral subspace $U_{0}$ is

$$
P_{0} u=x_{1} \varphi_{1}+x_{2} \varphi_{2}+x_{3} \varphi_{3} .
$$

The components of the vector $x=\left(x_{1}, x_{2}, x_{3}\right)$ are scalar products:

$$
x_{1}=\left(u, \Phi_{3}\right), \quad x_{2}=\left(u, \Phi_{2}\right), \quad x_{3}=\left(u, \Phi_{1}\right) .
$$

2. The equation on the neutral manifold in the normal form is

$$
\begin{aligned}
& \dot{x}_{1}=x_{2}+O\left(|x|^{2}\right), \\
& \dot{x}_{2}=x_{3}+O\left(|x|^{2}\right), \\
& \dot{x}_{3}=a x_{1} x_{2}+a_{1} x_{1} x_{3}+a_{2} x_{2}^{2}+a_{3} x_{2} x_{3}+a_{4} x_{3}^{2}+O\left(|x|^{3}\right) .
\end{aligned}
$$

3. The model system is

$$
\dot{x}_{1}=x_{2}, \quad \dot{x}_{2}=x_{3}, \quad \dot{x}_{3}=a x_{1} x_{2} .
$$

Here $a=2 \cdot\left(K_{2}\left(\varphi_{1}, \varphi_{2}\right)+A^{-1} K_{2} \varphi_{1}^{2}, \Phi_{1}\right)$.

Stability criteria [2]. The zero equilibrium of the system (2.1) is unstable if $a \neq 0$.

\section{The critical case of a double zero eigenvalue and simple pair eigenvalues on the imaginary axis}

1. The neutral spectrum is $\sigma_{0}(A)=\left\{0^{2}, \pm i \omega\right\}$. The eigenvalues are 0 of multiplicity 2 , which corresponds to the two-dimensional Jordan block and a simple pair $\pm i \omega(\omega>0)$. The corresponding generalized eigenvectors of operators $A$ and $A^{*}$ are $\varphi_{1}, \varphi_{2}, \varphi, \varphi^{*}$ and $\Phi_{1}=L(0), \Phi_{2}, \Phi, \Phi^{*}:$

$$
\begin{gathered}
A \varphi_{1}=0, \quad A \varphi_{2}=\varphi_{1}, \quad A \varphi=i \omega \varphi, \quad A \varphi^{*}=-i \omega \varphi^{*}, \\
A^{*} \Phi_{1}=0, \quad A^{*} \Phi_{2}=\Phi_{1}, \quad A^{*} \Phi=-i \omega \Phi, \quad A^{*} \Phi^{*}=i \omega \Phi^{*} \\
\left(\varphi_{1}, \Phi_{2}\right)=\left(\varphi_{2}, \Phi_{1}\right)=(\varphi, \Phi)=\left(\varphi^{*}, \Phi^{*}\right)=1 .
\end{gathered}
$$

The projector on the neutral subspace $U_{0}$ is

$$
P_{0} u=x_{1} \varphi_{1}+x_{2} \varphi_{2}+z \varphi+z^{*} \varphi^{*} .
$$

The components $x_{1}, x_{2}, z$ and $z^{*}$ of the vector $v_{0}=\left(x_{1}, x_{2}, z, z^{*}\right)$ are scalar products:

$$
x_{1}=\left(u, \Phi_{2}\right), \quad x_{2}=\left(u, \Phi_{1}\right), \quad z=(u, \Phi), \quad z^{*}=\left(u, \Phi^{*}\right) .
$$


2. The equation on the neutral manifold is

$$
\begin{aligned}
\dot{x}_{1} & =x_{2}+O\left(\left|v_{0}\right|^{2}\right), \\
\dot{x}_{2} & =b x_{1} x_{2}+x_{1} \operatorname{Re}\left[b_{1} z\right]+x_{2} \operatorname{Re}\left[b_{2} z\right]+b_{3}|z|^{2}+O\left(\left|v_{0}\right|^{3}\right), \\
\dot{z} & =i \omega z+O\left(\left|v_{0}\right|^{2}\right) .
\end{aligned}
$$

3. The model system is

$$
\dot{x}_{1}=x_{2}, \quad \dot{x}_{2}=b x_{1} x_{2}, \quad \dot{z}=i \omega z .
$$

Here $b=2 \cdot\left(K_{2}\left(\varphi_{1}, \varphi_{2}\right)+A^{-1} K_{2} \varphi_{1}^{2}, \Phi_{1}\right)$.

Stability criteria [2]. The zero equilibrium of the system (3.1) is unstable if $b \neq 0$.

\section{The critical case of simple zero and imaginary pair eigenvalues}

1. The neutral spectrum is $\sigma_{0}(A)=\{0, \pm i \omega\}$. The eigenvalues are simple 0 and pair $\pm i \omega$ $(\omega>0)$. The corresponding generalized eigenvectors of operators $A$ and $A^{*}$ are $\varphi_{1}, \varphi, \varphi^{*}$ and $\Phi_{1}=L(0), \Phi, \Phi^{*}$ :

$$
\begin{gathered}
A \varphi_{1}=0, \quad A \varphi=i \omega \varphi, \quad A \varphi^{*}=-i \omega \varphi^{*}, \quad A^{*} \Phi_{1}=0, \quad A^{*} \Phi=-i \omega \Phi, \quad A^{*} \Phi^{*}=i \omega \Phi^{*}, \\
\left(\varphi_{1}, \Phi_{1}\right)=(\varphi, \Phi)=\left(\varphi^{*}, \Phi^{*}\right)=1 .
\end{gathered}
$$

The projector on the neutral subspace $U_{0}$ is

$$
P_{0}=x \varphi_{1}+z \varphi+z^{*} \varphi^{*}
$$

The components $x, z$ and $z^{*}$ of the vector $v_{0}=\left(x, z, z^{*}\right)$ are scalar products

$$
x=\left(u, \Phi_{1}\right), \quad z=(u, \Phi), \quad z^{*}=\left(u, \Phi^{*}\right) .
$$

2. The equation on the neutral manifold in the normal form is

$$
\begin{aligned}
& \dot{x}=b_{0}|z|^{2}+b_{1} x|z|^{2}+b_{2} x^{2}|z|^{2}+b_{3}|z|^{4}+O\left(\left|v_{0}\right|^{5}\right), \\
& \dot{z}=i \omega z+D_{1} x z+D_{2} x^{2} z+D_{0} z|z|^{2}+O\left(\left|v_{0}\right|^{4}\right) .
\end{aligned}
$$

3.1. The model system is

$$
\dot{x}=b_{0}|z|^{2}, \quad \dot{z}=i \omega z+D_{1} x z
$$

Here

$$
b_{0}=2 \cdot\left(K_{2}\left(\varphi, \varphi^{*}\right), \Phi_{1}\right), \quad D_{1}=2 \cdot\left(K_{2}\left(\varphi_{1}, \varphi\right), \Phi\right), \quad d_{1}=\operatorname{Re} D_{1} .
$$

Stability criteria [2]. The zero equilibrium of the system (4.1) is unstable if $b_{0} d_{1}>0$ and stable if $b_{0} d_{1}<0$.

3.2. Degeneration $d_{1}=0$. The model system is

$$
\dot{x}=b_{0}|z|^{2}, \quad \dot{z}=i \omega z+D_{2} x^{2} z .
$$


Here

$$
\begin{gathered}
D_{2}=\left(H_{2}, \Phi\right), \quad H_{2}=2 K_{2}\left(\varphi, p_{1}\right)+4 K_{2}\left(\varphi_{1}, p_{2}\right)+3 K_{3}\left(\varphi_{1}^{2}, \varphi\right)-2 D_{1} p_{2}, \\
p_{2}=\frac{1}{2}(i \omega I-A)^{-1}\left(2 K_{2}\left(\varphi_{1}, \varphi\right)-D_{1} \varphi\right), \quad d_{2}=\operatorname{Re} D_{2} .
\end{gathered}
$$

Stability criteria [2]. Let $d_{1}=0$. The zero equilibrium (4.1) is stable if $d_{2}<0$ and unstable if $d_{2}>0$.

3.3. Degeneration $b_{0}=0$. The model system is

$$
\dot{x}=b_{1} x|z|^{2}+b_{3}|z|^{4}, \quad \dot{z}=i \omega z+D_{1} x z+D_{2} x^{2} z+D_{0} z|z|^{2} .
$$

Stability criteria [2]. Let be $b_{0}=0$ and $d_{1} \neq 0$. The zero equilibrium of the system (4.1) is stable under one of the conditions
a) $k_{1}<0, \quad k_{2}<0$,
b) $k_{1}>0, \quad k_{3}<0$

and unstable if $k_{2}>0$, or inequalities $k_{1}>0, k_{3} \geqslant 0$ are valid.

Here

$$
k_{1}=2 d_{0}+b_{1}, k_{2}=2 b_{3} d_{1}-2 d_{0} b_{1}, k_{3}=k_{1}^{2}+4 k_{2}, d_{0}=\operatorname{Re} D_{0} .
$$

The coefficients $b_{1}, b_{3}$ and $D_{0}$ are a scalar product:

$$
b_{1}=\left(h_{1}, \Phi_{1}\right), \quad b_{3}=\left(h_{3}, \Phi_{1}\right), \quad D_{0}=\left(H_{0}, \Phi\right) .
$$

The real vectors $h_{1}, h_{3} \in \mathbb{R}^{n}$ are defined by the equalities

$$
\begin{aligned}
h_{1} & \left.=8 \operatorname{Re}\left[K_{2}\left(\varphi, p_{2}^{*}\right)\right]+4 K_{2}\left(\varphi_{1}, p_{4}\right)+6 K_{3}\left(\varphi_{1}, \varphi, \varphi^{*}\right)\right)-4 \operatorname{Re}\left[D_{1} p_{4}\right]-2 b_{0} p_{1}, \\
h_{3} & =12 \operatorname{Re}\left[K_{2}\left(\varphi, q_{4}^{*}\right)\right]+2 K_{2}\left(p_{3}, p_{3}^{*}\right)+4 K_{2} p_{4}^{2}+6 \operatorname{Re}\left[K_{3}\left(\varphi^{2}, p_{3}^{*}\right)\right]+ \\
& +12 K_{3}\left(\varphi, \varphi^{*}, p_{4}\right)+6 K_{4}\left(\varphi^{2}, \varphi^{* 2}\right)-4 \operatorname{Re}\left[D_{0} p_{4}\right]-6 b_{0} q_{3} .
\end{aligned}
$$

The complex vector $H_{0} \in \mathbb{C}^{n}$ is defined by the equality

$$
H_{0}=3 K_{3}\left(\varphi^{2}, \varphi^{*}\right)+2 K_{2}\left(\varphi^{*}, p_{3}\right)+4 K_{2}\left(\varphi, p_{4}\right)-2 b_{0} p_{2} .
$$

The real vectors $p_{1}, p_{4}, q_{3} \in \mathbb{R}^{n}$ have the form

$$
\begin{aligned}
p_{1}= & -A^{-1} K_{2} \varphi_{1}^{2} \\
p_{4}= & -\frac{1}{2} A^{-1}\left(2 K_{2}\left(\varphi, \varphi^{*}\right)-b_{0} \varphi_{1}\right) \\
q_{3}= & -\frac{1}{6} A^{-1}\left(8 \operatorname{Re}\left[K_{2}\left(\varphi, p_{2}^{*}\right)\right]+4 K_{2}\left(\varphi_{1}, p_{4}\right)+6 K_{3}\left(\varphi_{1}, \varphi, \varphi^{*}\right)-\right. \\
& \left.-4 \operatorname{Re}\left[D_{1} p_{4}\right]-2 b_{0} p_{1}-b_{1} \varphi_{1}\right) .
\end{aligned}
$$

The complex vectors $p_{2}, p_{3}, q_{4} \in \mathbb{C}^{n}$ are given by the formulas

$$
\begin{aligned}
& p_{2}=\frac{1}{2}(i \omega I-A)^{-1}\left(2 K_{2}\left(\varphi_{1}, \varphi\right)-D_{1} \varphi\right), \\
& p_{3}=(2 i \omega I-A)^{-1} K_{2} \varphi^{2}, \\
& q_{4}=\frac{1}{3}(i \omega I-A)^{-1}\left(2 K_{2}\left(\varphi^{*}, p_{3}\right)+4 K_{2}\left(\varphi, p_{4}\right)+3 K_{3}\left(\varphi^{2}, \varphi^{*}\right)-D_{0} \varphi-2 b_{0} p_{2}\right) .
\end{aligned}
$$




\section{References}

[1] Yudovich, V. I., Cosymmetry, Degeneration of Solutions of Operator Equations, and the Onset of Filtration Convection, Math. Notes, 1991, vol.49, nos.5-6, pp.540-545; see also: Mat. Zametki, 1991, vol. 49, no. 5, pp. 142-148.

[2] Kurakin, L. G., Critical Cases of Stability. Converse of the Implicit Function Theorem for Dynamical Systems with Cosymmetry, Math. Notes, 1998, vol.63, nos. 3-4, pp. 503-508; see also: Mat. Zametki, 1998, vol. 63, no. 4, pp. 572-578.

[3] Kurakin, L. G. and Yudovich, V. I., Semi-Invariant Form of Equilibrium Stability Criteria in Critical Cases, J. Appl. Math. Mech., 1986, vol.50, no.5, pp.543-546; see also: Prikl. Mat. Mekh., 1986, vol. 50, no. 5, pp. 707-711.

[4] Pliss, V.A., A Reduction Principle in the Theory of Stability of Motion, Izv. Akad. Nauk SSSR Ser. Mat., 1964, vol. 28, no. 6, pp. 1297-1324 (Russian).

[5] Arnold, V.I., Additional Chapters of the Theory of Differential Equations, Moscow: Nauka, 1978 (Russian).

[6] Rumyantsev, V.V. and Oziraner, A.S., Motion Stability and Stabilization with Respect to Part of Variables, Moscow: Nauka, 1987 (Russian). 